\title{
Quieting the Flows in Valves Using Kinetic Energy Degraders
}

\author{
Michel Pluviose* \\ Chair of Turbomachines \\ Conservatoire national des Arts et Métiers, 292, rue Saint-Martin, 75141 Paris Cedex 03, France \\ michel_pluviose@numericable.fr
}

\begin{abstract}
Valves are equipment which throttle a fluid to adjust the flow rate. At the opening of a relief valve, the mass of fluid released has a very large kinetic energy which is dissipated in a complex way in the pipework downstream. The high pressure ratio at valve opening carries the fluid system away from its initial equilibrium. After traveling through some bifurcations and fluctuating zones, this fluid system becomes self-organized. Flows that are thus confronted with energy dissipation and instabilities are dangerous for installations. Current recommended practice consists in installing internal devices which limit the disturbances to acceptable values, by weakening the chaotic phenomena without eliminating them. The kinetic energy degrader presented in this paper is an original device. It makes it possible to avoid chaotic zones and massively degrades the kinetic energy contained in the fluid. As a result, the fluid reaches its final equilibrium much more quickly. Flows in valves are thus quieted and the facilities are therefore protected.
\end{abstract}

Keywords: degrader; non-linear; valve; worst action.

\section{Introduction}

The motive power embedded in valve flows, which sometimes reaches tens of megawatts, can become dangerous. According to the second law of thermodynamics, the kinetic energy contained in the fluid is irreversibly dissipated with an increase in disorder, which corresponds to entropy. Dissipative phenomena destroy kinetic energy by transforming it in a complex and often brutal way into vibratory energy, sound energy, etc., and then finally into thermal energy. It is the quality of the energy which is degraded and not its total quantity, which is preserved according to the first law of thermodynamics. One of the major insights of non-linear thermodynamics is that order is created far from initial equilibrium. Ordered structures are reached after the passage through chaotic zones.

Chaos theorists, the first of whom was Henri Poincaré, and more recently Ilya Prigogine (1986), René Thom (1983) and many others (Gilmore, 1993; Gleick, 1987; Manneville, 1990; Mandelbrot, 1995), have stirred physics up by questioning the determinism of the laws of motion. Prigogine in particular, during the 1970s, drew attention to the self-organized structures which arise far from equilibrium. He called them dissipative structures to associate the two ideas of order and waste (Prigogine \& Stengers, 1986). These dissipative structures are orderly creations which we can observe all around us, and which arouse our admiration.

Chaos physicists do not seem to have pursued their reflection beyond the observation and description of phenomena. However, we must try here to destroy this chaos which invades valves, because the dissipative structures and the instabilities that accompany them are too slow to disappear, and dangerously excite the structures.

Boltzmann showed that entropy is a measurement of the disorder which reigns in the molecular world (Prigogine \& Stengers, 1986; Prigogine \& Kondepudi, 1999). In the final state of equilibrium, after the degradation of kinetic energy, entropy reaches its maximum value.

Before reaching Boltzmann's state of maximum disorder, Prigogine says that it is initially necessary to go through a stage of dissipative structures which, by exchanging matter and energy with their surroundings, slow down the return to equilibrium. That is why planets, plants, etc., have a certain lifetime before dying out.

Supersonic jets in valves, which are too slow to disappear, are another example. For our safety, they must be destroyed.

Is it possible to quickly reach Boltzmann's maximum disorder while avoiding fluctuating zones and Prigogine's dissipative structures? Or put more simply: can we avoid chaos in valves?

The second law of thermodynamics states that: "energy is degraded ". In its application to valves, we express this by: "energy must be degraded ", to show our determination to act in order to amplify this dissipation not to undergo it.

Hereafter, a proactive solution is proposed which modifies the flow's molecular system by intensifying transport phenomena, so that the kinetic energy is converted directly into thermal energy. Chaos can thus be avoided with a massive creation of entropy.

\section{Principle of Least Action}

The principle of least action is based on the following assertion: "Nature dislikes exhausting itself " (Planck, 
1915). It covers many areas in physics such as quantum mechanics, analytical mechanics and of course classical mechanics. Friction is not taken into account. In these conditions, all natural phenomena can be reduced to a law of conservation and are reversible. The first law of thermodynamics, which states that energy is conserved, will be subsumed here under the principle of least action.

\section{Principle of Worst Action}

The second law of thermodynamics, which stipulates that energy is degraded, will, for the purposes of the present argument, be raised to the rank of principle of worst action (Pluviose, 2012). In any system, an engineer hunts out the main sources of irreversibilities and tries to eliminate them, or at least to minimize them so as to improve the efficiency of the installations (Bejan, 1982).

The approach hereafter will be radically different because it is necessary for our purposes to create many irreversibilities, and therefore much entropy or disorder.

This method, which may seem reprehensible at first sight, will prove to be useful and necessary. The principle of worst action will turn out to be particularly effective in the branch of physics, neglected by physicists, where large amounts of energy must be necessarily degraded. While all the systems in classical mechanics, among which the kinetic theory of gases, are symmetrical with respect to time and are therefore reversible, the second law, with its irreversibilities, imposes the existence of the arrow of time.

Although Clausius contributed to the development of the kinetic theory of gases, he wished to keep the principles of thermodynamics free from any molecular reference. As well as Sadi Carnot, he thus made of it a purely macroscopic discipline. But Boltzmann was not satisfied with the abstract nature of the second law of thermodynamics. He therefore tried to write this second law on the mechanical basis of the movement of molecules, given that these objects move according to Newton's reversible law. An absolutely amazing result ensued: the reversible phenomena of the microscopic world, when entering our macroscopic world made up of huge numbers of molecules, are transformed into irreversible processes with an increase in entropy. Because of their fabulous number, it is absolutely impossible to track the movements of molecules in detail and it is this absence of complete predictability, this uncertainty, which imposes a direction on time.

\section{Duality between Least Action and Worst Action}

These two principles live together, sometimes on good terms when one of them keeps quiet in relation to the other, but often in conflict when one of them becomes the dominant partner. This is particularly noticeable in the complexity which arises when changing the scale of observation from that of the microscopic world to that of our macroscopic world. These two worlds coexist within us and around us. Though independent in appearance, they are in fact deeply entangled, and constantly interfere, squabble and tussle with each other. One of them is on the human scale, while the other much smaller one is capable of organizing itself and can become formidable. The microscopic and macroscopic worlds are radically different:

- The microscopic world is discontinuous, reversible, linear; it is governed by the principle of least action.

- The macroscopic world is continuous, irreversible, nonlinear. It is the domain where the principle of worst action reigns and which becomes particularly apparent when the irreversibilities increase.

\section{Disturbances in a Sudden Enlargement}

Borda, an acute observer of nature, was particularly interested by the structures of water flowing from a faucet. When these flows are channeled downstream in a pipe, it becomes much more difficult to capture their fundamental physical nature because the flow loses part of its freedom while interfering with the sidewalls: the jet is said to be confined (Blake, 1986). Borda showed that energy dissipations in such a device are directly related to the kinetic energy of the jet. Let us consider a two-dimensional sudden enlargement, as shown in Figure 1, through which a compressible fluid now passes.

Because of the limitations of Schlieren visualization, only a 2D representation of a sudden enlargement is shown here (Fabri \& Siestrunck, 1955). Starting from equilibrium, and by increasing the pressure ratio which is chosen as the control parameter, the flow passes through several zones.
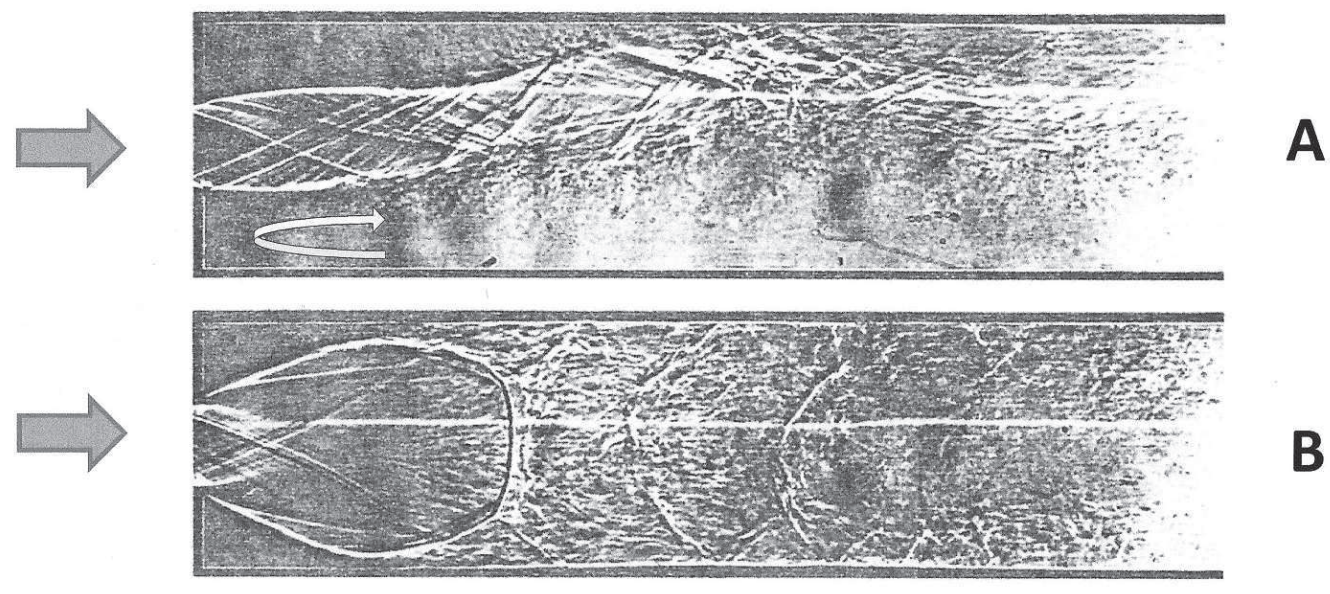

Figure 1. Schlieren visualizations obtained before (A) and after (B) spatiotemporal chaos in a two-dimensional sudden enlargement. 
At equilibrium, only the principle of least action reigns. Entropy is zero and phenomena are reversible, so that mathematics and physics are in perfect harmony.

Close to equilibrium, therefore for a very low pressure ratio, we are in the domain of linear thermodynamics. Agitation in the molecular world leads to transport phenomena which are irreversible processes. In particular, the molecular transport of momentum produces the macroscopic effect called viscosity. The energy dissipations which result from these phenomena increase entropy and reveal the arrow of time: there is now a past and a future. In this linear zone, the flows are laminar and all the fluctuations regress under the influence of viscosity. The differential equations which govern the flow have a single solution. Close to equilibrium, the principle of least action dominates the principle of worst action.

Farther from equilibrium and on reaching a bifurcation point, one enters the non-linear domain where the regime becomes turbulent. Flows are irregular and complex but it is not complete disorder. Although the system seems to be random, it is possible to detect the presence of order in a strange attractor, which makes the system deterministic (Gleick, 1987). The Navier-Stokes equations, which describe this situation, are non-linear in many aspects (De Groot, 1951; Baker et al., 1997; Lebon et al., 2008), which introduces the seeds of chaos into the flow. Physics and mathematics gradually move apart.

By increasing the pressure ratio, one reaches the supersonic regime where, after a second bifurcation point, the jet flattens itself on one wall or on the other in a totally unpredictable fashion, as shown in Figure 1A.

Chance determines the future of the entire macroscopic system. This attachment is quite sudden but stable. The explanation for this phenomenon is to be sought in the jet's capacities of stability (Pluviose, 1991).

Due to viscous effects, the jet, which is assumed to be centered, accelerates the peripheral fluid layers. The mass thus transported is renewed by the fluid which comes from downstream. Because the two cavities which frame the jet are independent of each other, the smallest random perturbation destabilizes the system. For the cavity connected to the outside in Figure 1A, there is an exchange of both energy and matter with the external environment. In this case, and because the external reserves of energy and matter are large enough to remain in a permanent state, the system goes towards a constant state other than that of equilibrium: it attains a steady state of non-equilibrium. As the entropy of such an open system can decrease, this system evolves then to greater order.

The central jet swells when one moves further away from equilibrium by increasing the pressure ratio more and more.

From another bifurcation point, the jet becomes unstable, causing longitudinal and transverse oscillations of the fluid stream: it is spatiotemporal chaos. The violent instabilities observed are due to the enlargement of the jet, which reduces the cross-section for the passage of the return fluid feeding the dead corner. This feedback is responsible for this spatiotemporal chaos accompanied by transient phenomena similar to intermittences between two regimes that have become unstable as a result of conflicting influences of the same magnitude.

After a sudden priming for even higher pressure ratios, the jet is now recentered as shown in Figure 1B. Oblique shock wave systems now happen and increasingly invade the downstream cavity before the fluid is evacuated in a very turbulent subsonic regime.

\section{Escape from Chaos and Dissipative Structures}

Chaos theory emerged with the development of computers, it is thus recent and although its wellfoundedness has been proven in many areas of life, industry, which is still strongly anchored in determinism, has been reluctant to acknowledge it. And yet, in the sudden enlargement which is an industrial device, it is clearly chaos which manifests itself, and with it dissipative structures. For a system far from equilibrium, two very awkward problems concerning the waste of kinetic energy arise:

- first, the spatiotemporal chaos which destabilizes the entire flow,

- then, the dissipative structures which can introduce a degree of order into the system.

\section{The Kinetic Energy Degrader or Vistemboir}

The dissipative structures, which follow the chaotic zones, are cumbersome and hinder the return to equilibrium. They are incapable of degrading kinetic energy quickly and on the spot. In the present case, these dissipative structures are undesirable and it is therefore necessary to destroy them. But how can this be done?

When Borda experimented with water in his demonstrations, at speeds similar to those of the tranquil flow of a river, he ran no risk of exciting the structures of his installation. The manner in which kinetic energy in the fluid was destroyed was therefore not Borda's concern, but it is ours here and now. Nowadays, devices such as perforated plates, the modern descendants of Borda's sudden enlargement, have to degrade kinetic energy which has a considerable motive power (Richard \& Pluviose, 1991). To give an idea of the orders of magnitude, powers of 10 megawatts can be encountered. Abrupt fluctuations in fluid pressure can stress the structures, fatigue them and finally deform them. Before that, little by little, cracks start to appear. Since the kinetic energy of the fluids has not been destroyed, they degrade this energy themselves, but in ways that can severely damage the installation. Is it wise to allow flows to behave in such an uncontrolled manner, leading to spatiotemporal chaos, when we know that the motive power to be degraded can reach such magnitudes? Since there remains a certain degree of order in a dissipative structure, it does not degrade sufficient kinetic energy. It is thus necessary to invent another structure which could be called: kinetic energy degrader or, put more briefly, vistemboir. This device is the material element which concretely implements the principle of worst action.

The simplest vistemboir consists of a two-dimensional supersonic expansion capable of producing intense mixing. Supersonic bell-shaped nozzles are laid out on both sides of a central supersonic jet. They are fed in the same conditions as the central jet and built in such a way that a recompression shock wave exists before their flow penetrates the mixing 
area. Behind this shock wave, the flow is therefore subsonic, as shown in Figure 2.

In the mixture zone, considerable differences in speed are created between the central supersonic flow and the peripheral subsonic flows.

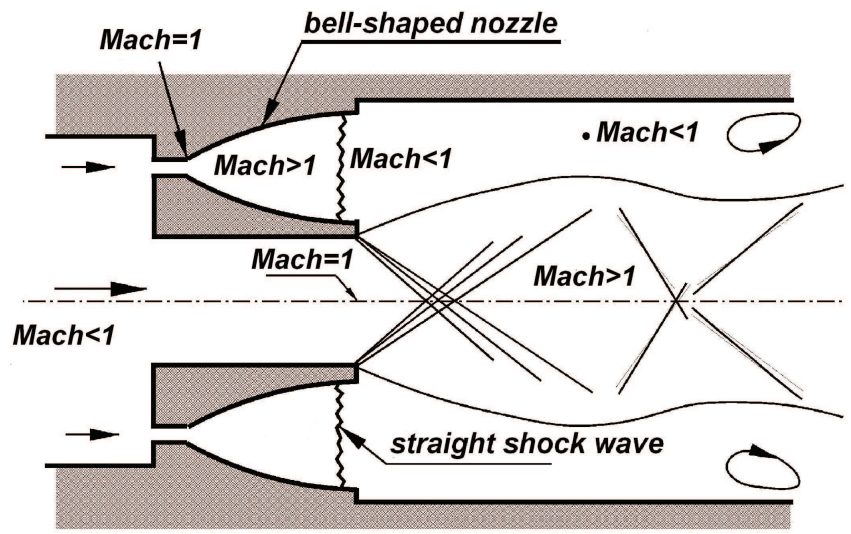

Figure 2. Kinetic energy degrader: the simplest vistemboir.

Since this difference in speed is greater than the average speed of the molecules, the molecules' movement must be strongly affected and the exchanges of momentum are very intense. The fluid system is stabilized and in addition the dissipation of kinetic energy is both rapid and much greater than in a simple sudden enlargement. The effect of the vistemboir on the flows is dreadful. So much the better! The vistemboir goes against the usual objectives sought in applying fluid mechanics (Pluviose, 1989).

\subsection{Kinetic Energy Degrader for Control Valves}

When we solve the partial differential equations that describe flows, we discover a curious fact. In the supersonic regime, these equations are hyperbolic and lead to some real characteristics at each point in the field.
(A)

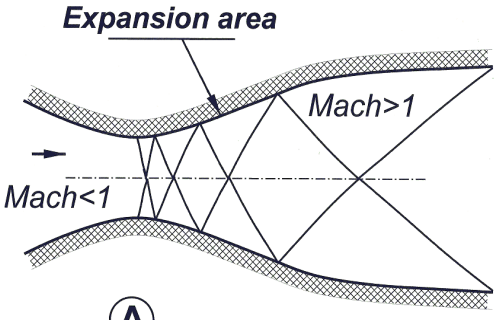

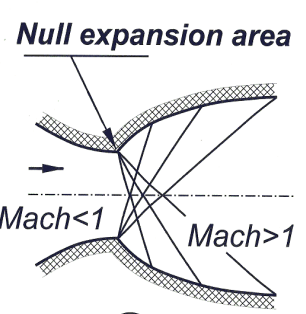

(B)
Figure 3. Conventional (A) and Bell-shaped nozzles (B).

In the more familiar subsonic regime, the equations are elliptical and lead to imaginary characteristics. The fact that the supersonic regime is relatively easy of access is a pleasant surprise that nature springs on us.

The method conceived by studying sudden enlargements can be adapted to improve the operation of valves. In an initial approximation, the flows in the two types of nozzles, shown in Figure 3, follow the principle of least action because in the absence of shock waves, the friction losses are zero. But when the nozzles are brought closer to each other, everything will change. Indeed, let us take these two nozzles to build a multi-structured device in which each nozzle has a given thickness. In such a sandwich structure, the pressures, velocities, etc., of each layer are very different. For its use in control valve, alveoli shaped like bell-shaped nozzles are hollowed out in the recovery nozzle of the valve. These alveoli are distributed in the nozzle and separated from one another by conventional nozzles, as shown in Figure 4. Different flows thus occur, depending on the azimuthal penetration in the valve.
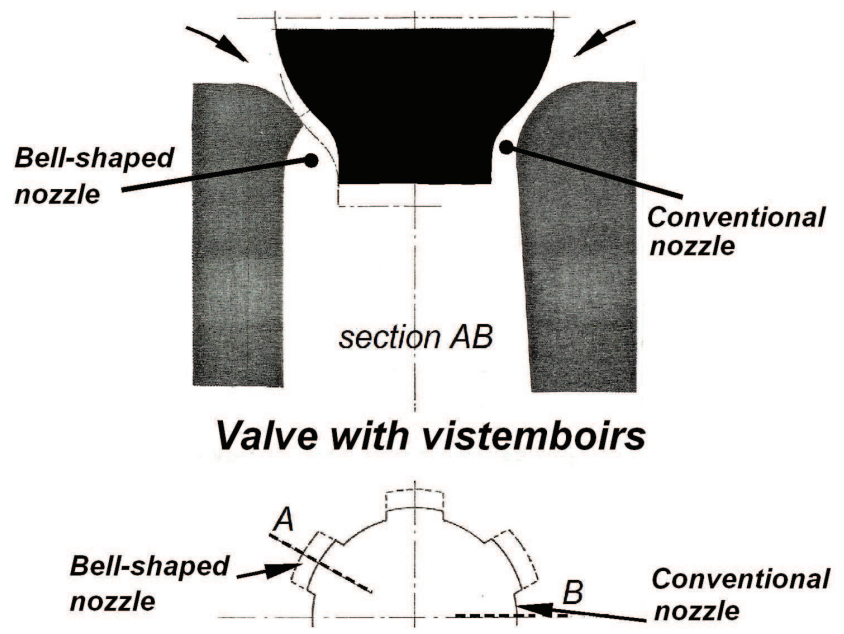

Figure 4. Control valve with vistemboirs.

While the fluid is homogeneous upstream, it is forced to expand suddenly from the throat either in a bell-shaped nozzle or in a conventional nozzle, as shown in Figures 4 and $5 \mathrm{~B}$. The stratified structure which is imposed on the flow forces it to immediately abandon the principle of least action and to activate the principle of worst action, entailing intense mixing throughout the mass of fluid. Not a single particle is identical to its neighbor, neither upstream nor downstream, to its left or to its right. Non-linear transport phenomena undertake their irreversible and degrading activity.

An opportunity offered by nature has been seized because it was possible to diversify supersonic flows before unexpectedly setting them side by side, thereby creating an indescribable brawl in the world of very small particles. The molecular system is disturbed and new phenomena, which are not yet understood, suddenly act to rapidly increase the creation of entropy. This enables us, in our macroscopic world, to impose a behavior on the microscopic world. The physics of these phenomena, which restores calm to our world, remains to be investigated in greater depth: it implies giving up the dissipative structures of Prigogine in favor of Boltzmann's disorder. Entropy production is very important and Boltzmann's relation is almost verified. In this adventure, disorder has been organized in the microscopic world to avoid chaos in our macroscopic world.

The behavior of the new geometry equipped with vistemboirs and that of the reference geometry can be compared by recording the unsteady forces on the stem of a valve. The results are recorded during a gust test. In this type of test, the full range of pressure ratios for a given degree of lift can be obtained. 

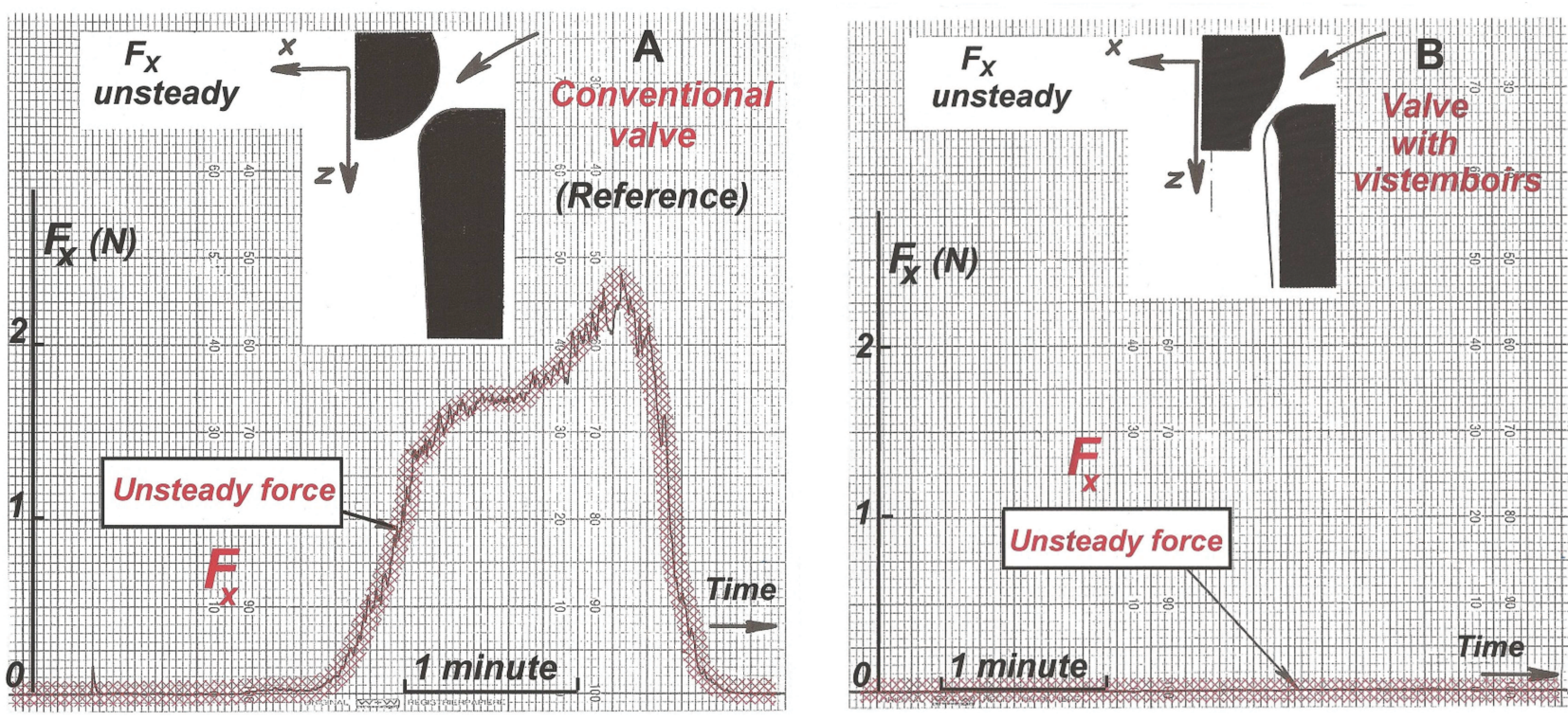

Figure 5. Quieting the flows in control valves with vistemboirs (Figure is in color in online version of paper).

The result is spectacular, as shown in Figure 5: in the valve with vistemboirs, the signal delivered by the sensor is extremely low compared to the signal with the conventional valve. When the vistemboir is applied, this causes an immediate reduction in all the fluctuating features of the fluid system, as shown in Figure 5. The most significant result is the fact that this decisive improvement was obtained from the very first attempt, which is undoubtedly the best proof of the efficiency of this solution. Flows in control valves are finally subdued!

\subsection{Kinetic Energy Degrader for Safety Valves}

Safety valves, from Papin's time until today, have undergone many significant changes in their effects on flows. The main alteration, for present purposes, is the implementation of a collector to recover the fluid expelled. All the motive power contained in the fluid, which was previously released into the atmosphere, is now forced to dissipate in the reduced space of a piping (Chabane et al., 2009). In view of the amplitude of the free jet which escapes from a safety valve, as shown in Figure 6 for example, it is clear that the flow is severely hampered by the confined space in which it now has to expand downstream. The phenomena are too complicated for me to claim to be able to describe them. All that we can be sure of is that the flow will manage to degrade the motive power of the jet as best suits its own interests, quirks, and whims, without worrying in the slightest about ensuring our safety, our environment, or our installations.

During the opening of a valve, the disturbances caused by the motive power of the jet can deform the structure and sometimes block closing. In other situations where a valve is closed again, it is often no longer leakproof at closure. The mechanical effects of such chaotic flows on structures are poorly understood. In a supersonic flow subjected to such illtreatment, the slightest detail can lead to disaster. All these valves are sick and even if all of them do not die, they must all be cured. The principle of worst action makes essential improvements to their operation (Pluviose, 2012).

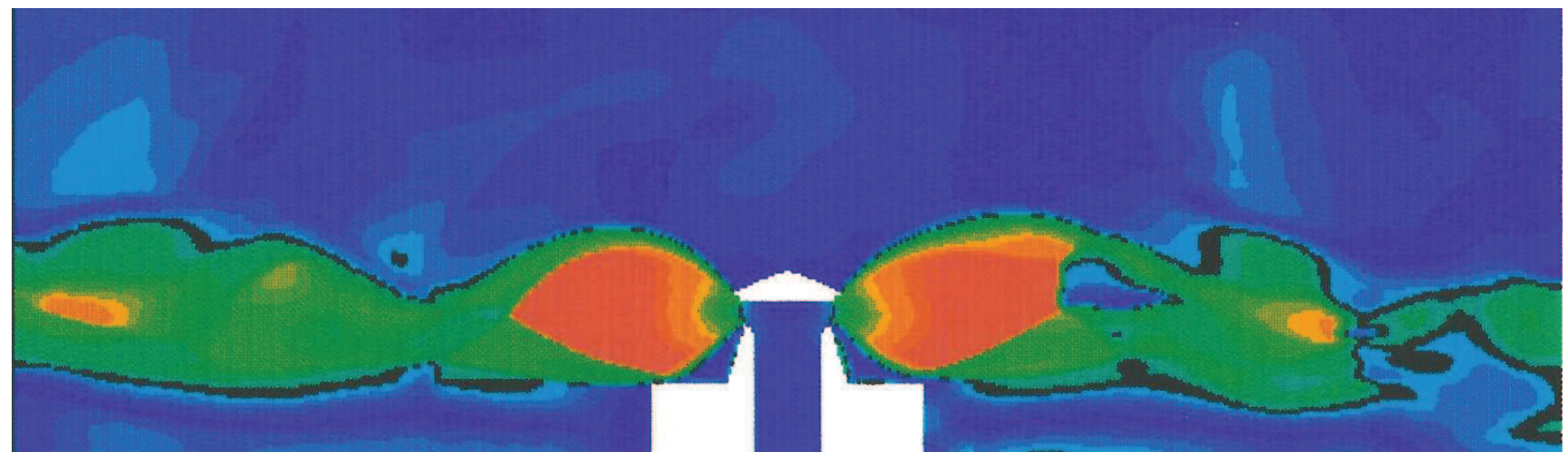

Figure 6. Free jet at the exhaust of Denis Papin's safety valve. The equations of inviscid flows (no friction) were solved numerically with a shock capturing method. Calculations kindly made by the Directorate of Studies and Research at Electricité of France (EDF) (Figure is in color in online version of paper). 
Everybody knows that these devices led to very serious accidents. Research then was undertaken to investigate these problems (Pluviose, 1989; Watremetz \& Pluviose, 1998). The improvements that were achieved in the behavior of valves by implementing the new geometry reassured many manufacturers, all over the world, who were faced with dangerous instabilities in their machines. When the results were published in 1989, however, their deeper meaning was not grasped at the time.

\section{Physics Challenges Mathematics}

Navier-Stokes equations take viscous effects into account in a zone close to equilibrium. For example, it's the domain limited by Stokes' law, which linearly connects the stress field with the velocity field in a fluid flow. These equations still contain major challenges. The farther we are from equilibrium, the greater the difference between physics and mathematics. Little by little, continuum mechanics gives way to discontinuum mechanics. In the present study, where the devices produce friction in abundance, it becomes increasingly complex to derive and solve the requisite equations. This is an extreme case and a major challenge that physics poses to mathematics.

\section{Epilogue}

At a time when everyone is attempting to avoid wasting energy, it is essential to realize that in certain situations, we must degrade it. Among these irreversibilities we find for example, the jet of water flowing from a tap, a low power jet which deserves no further comment. Or the supersonic jet in safety valves, with a motive power that is so great nowadays that these valves have become unsafe and can cause damage, incidents and accidents that are often global in scope. Without realizing it, we have allowed chaos to appear in safety devices that were designed to protect people, the environment and the installations. In these devices that have a strong motive power embedded in the flow, it is necessary to force the flow to degrade its kinetic energy quickly and on the spot, in an entropic forcing device that creates massive disorder. The only principle which should be applied in this case is the principle of worst action; the principle of least action must be gagged, because it would result in flow fluctuations with dangerous vibrations for the structures, then the emergence of self-organization that is insufficiently dissipative. The macroscopic world then has to provide enough instructions by imposing a very complex structure on the microscopic world so that it complies with Boltzmann's view and not with Prigogine's order through fluctuations. The use of vistemboirs ensures not only a significant and useful waste of kinetic energy but also a correct stabilization of the flows. They force the billions and billions of molecules in the microscopic world to abandon their inclination to form marvellous but dangerous mixtures of determinism and chance.

Thus the walls guiding the fluid regain their serenity.

\section{Acknowledgments:}

The author wishes to thank the French manufacturers of power plants and Electricity of France for giving him their fullest support and cooperation.

\section{References:}

Baker, G., McRobie, F. A., Thompson, J. M. T. (1997). Implications of Chaos Theory for Engineering Science. Proc. Instn. Mech. Engrs., 211, 349-363.

Bejan, A. (1982). Entropy Generation through Heat and Fluid Flow. New York, NY: Wiley-Interscience.

Blake, W. K. (1986). Mechanics of Flow-Induced Sound and Vibration. New York, NY: Academic Press Inc.

Chabane, S., Plumejault, S., Pierrat, D., Couzinet, A., Bayart, M. (2009). Vibration and chattering of conventional safety relief valve under built up back pressure. IAHR Czech., 3, 281-294.

De Groot, S.R. (1951). Thermodynamics of Irreversible Processes. Amsterdam, The Netherlands: North-Holland Publishing Company.

Fabri, J., Siestrunck, R. (1955). Divers régimes d'écoulement dans l'élargissement brusque d'une veine supersonique. Rev. Gén. Sci. Appl., 2, 229-237.

Gilmore, R. (1993). Catastrophe Theory for Scientists and Engineers. New York, NY: Dover Publications Inc.

Gleick, J. (1987). Chaos. New York, NY: The Viking Press.

Lebon, G., Jou, D., Casas-Vasquez, J. (2008). Understanding Non-Equilibrium Thermodynamics. Berlin, Germany: Springer-Verlag.

Mandelbrot, B. (1995). Les Objets Fractals, (4th ed.). Paris, France: Flammarion.

Manneville, P. (1990). Dissipatives Structures and Weak Turbulence. London, England: Academic Press.

Planck, M. (1915). The Principle of Least Action. Retrieved from www.ias.ac.in / resonance / February 2008 / p198-207.pdf.

Pluviose, M. (1989). Stabilization of Flow through SteamTurbine Control Valve. ASME Journal of engineering for Gas Turbines and Power, 111, 642-646.

Pluviose, M. (1991). Instabilities of Flow in Sudden Enlargements. Winter ASME, 128, 291-300.

Pluviose, M. (2012). Rescuing Valves in Distress. Self-published at www.lulu.com.

Prigogine, I., Stengers, I. (1986). La Nouvelle Alliance. Paris, France: Gallimard.

Prigogine, I., Kondepudi, D. (1999). Thermodynamique. Paris, France: Odile Jacob.

Richard, J.-M., Pluviose, M. (1991). Aerodynamic Instabilities in Governing Valves of Steam Turbines. iMechE London. 3, 93-102.

Thom, R. (1983). Paraboles et Catastrophes. Paris, France: Flammarion.

Watremetz, M., Pluviose, M. (1998). Des soupapes plus tranquilles. Cetim-Informations, 160, 47-48. 